\title{
A result on total coloring of circulant graphs
}

\author{
Mauro N. Alves Junior ${ }^{1}$, Diana Sasaki ${ }^{1}$ \\ ${ }^{1}$ IME, Rio de Janeiro State University, Brazil
}

\begin{abstract}
In this work we determine that all members of an infinite family of 4-regular circulant graphs are Type 1.
\end{abstract}

Resumo. Neste trabalho, nós provamos que todos os membros de uma família infinita de grafos circulantes 4-regulares são Tipo 1.

\section{Introduction}

Total colorings combine the vertex and edge colorings by coloring both vertices and edges of a graph $G$ so that adjacent elements (vertices and edges) have different colors. A $k$-total coloring of a graph $G$ is an assignment of $k$ colors to the elements of $G$ such that adjacent elements have different colors. The total chromatic number $\chi^{\prime \prime}(G)$ is the smallest integer $k$ for which $G$ has a $k$-total coloring. Clearly, $\chi^{\prime \prime}(G) \geq \Delta+1$, and the Total Coloring Conjecture (TCC) states that for any simple graph $G, \chi^{\prime \prime}(G) \leq \Delta+2$, where $\Delta$ is the maximum degree of $G$ [Behzad 1965, Vizing 1968]. Graphs with $\chi^{\prime \prime}(G)=\Delta(G)+1$ are said to be Type 1 , and graphs with $\chi^{\prime \prime}(G)=\Delta(G)+2$ are said to be Type 2 . The TCC has been verified for several classes of graphs.

A circulant graph $C_{n}\left(d_{1}, d_{2}, \cdots, d_{l}\right)$ with $1 \leq d_{1}<\cdots<d_{l} \leq\left\lfloor\frac{n}{2}\right\rfloor$ has vertex set $V=\left\{v_{0}, v_{1}, \cdots, v_{n-1}\right\}$ and edge set $E=\bigcup_{i=1}^{l} E_{i}$ where $E_{i}=\left\{e_{0}^{i}, e_{1}^{i}, \cdots, e_{n-1}^{i}\right\}$ and $e_{j}^{i}=\left(v_{j}, v_{j+d_{i}}\right)$ where the indices of the vertices are considered modulo $n$. An edge of $E_{i}$ is called edge of length $d_{i}$. Examples of circulant graphs include the cycle graphs $C_{n} \simeq C_{n}(1)$ and the complete graphs $K_{n} \simeq C_{n}\left(1,2, \cdots,\left\lfloor\frac{n}{2}\right\rfloor\right)$, and their total chromatic number are well known [Yap 1996]. Furthermore, the total chromatic number of the cubic circulant graphs (which are of the form $C_{2 n}(d, n)$ ) was determined in 2004 [Hackmann and Kemnitz 2004]. The well known Möbius Ladder graphs are the cubic circulant graphs $C_{2 n}(1, n)$, and they are known to be Type 2 [Chetwynd and Hilton 1988]. In 2008, it was proved that every 4-regular circulant graph, for any positive integer $p, C_{5 p}(1, k)$ is Type 1 for $k<5 p / 2$ with $k \equiv 2 \bmod 5$ or $k \equiv 3 \bmod 5$; and $C_{6 p}(1, k)$ is Type 1 for $p \geq 3$ and $k<3 p$ with $k \equiv 1 \bmod 3$ or $k \equiv 2 \bmod 3$ [Khennoufa and Togni 2008].

Another infinite family of circulant graphs which have been extensively studied in the literature is the power of cycles, denoted by $C_{n}^{k}$, and consisting of a cycle $C_{n}$ with additional edges between any pair of vertices of distance at most $k$. It was conjectured that $C_{n}^{k}$, with $2 \leq k \leq\lfloor n / 2\rfloor$, is Type 2 if and only if $n$ is odd and $k<n / 3-1$ [Campos 2006]. This implies that for each $k \geq 2$, there is a finite number of Type 2 graphs. This conjecture has been proved when $k=2$ and in the same work, she proved that the TCC holds for $n$ even and for $C_{n}^{3}$ and $C_{n}^{4}$ [Campos 2006]. Recently Campos' conjecture has been proved for $C_{n}^{3}$ and $C_{n}^{4}$ [Zorzi 2019].

In this work, we determine the total chromatic number of another family of circulant graphs. The technique uses total colorings of the well known Ladder graphs, also 
named as Prism graphs $G(n, 1)$. For these graphs it was proved that they are all Type 1, except for $G(5,1)$ which is Type 2 [Chetwynd and Hilton 1988].

\section{Preliminaries}

A semigraph is a triple $B=(V, E, S)$ where $V$ is the set of vertices of $B, E$ is a set of edges having two distinct endpoints in $V$, and $S$ is a set of semiedges having one endpoint in $V$. We denote an edge having endpoints $v$ and $w$ by $v w$ and a semiedge having endpoint $v$ as $v$. When vertex $v$ is an endpoint of $e \in E \cup S$ we say that $e$ is incident to $v$. Two elements of $E \cup S$ incident to the same vertex, or two vertices incident to the same edge, are called adjacent. In this work, we are mainly interested in graphs and semigraphs such that there are exactly four elements (edges and/or semiedges) incident to every vertex. These are called 4-regular graphs and 4-regular semigraphs, respectively. Notice that a $k$-total coloring of a semigraph $B$ is an assignment of $k$ colors to the edges, semiedges and vertices of $B$ such that adjacent elements have different colors.

Every 4-regular circulant graph is denoted by $C_{n}(a, b)$, with $1 \leq a<b<n / 2$. We investigate the circulant graphs that are a subclass of the class of graphs $C_{n}(a, b)$, with $a$ mutiple by $n$ or $b$ multiple by $n$. All graphs of this class have the Ladder graphs $G\left(\frac{n}{a}, 1\right)$ (or $G\left(\frac{n}{b}, 1\right)$ ) as a subgraph (see Figure 1). We use appropriate total colorings previously obtained for this class [Chetwynd and Hilton 1988] to construct 5-total colorings of Theorem 1.
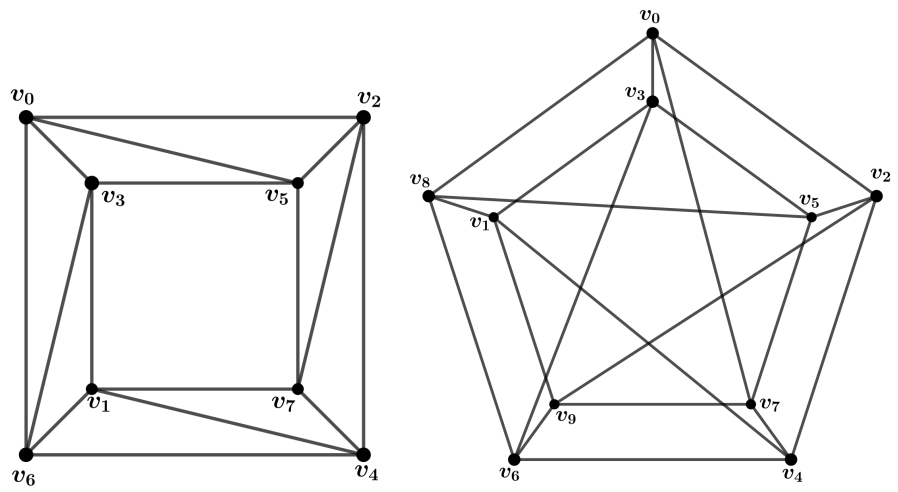

Figure 1. The 4-regular circulant graphs $C_{8}(2,3)$ and $C_{10}(2,3)$ with the Ladder graphs $G(4,1)$ and $G(5,1)$ as subgraphs, respectively.

\section{An infinite family of Type 1 circulant graphs}

The main result of this work is presented below.

Theorem 1. Let $C_{n}(2 k, 3)$ be a 4-regular connected circulant graph. The graph $C_{n}(2 k, 3)$ is Type 1 for $n=(8 \mu+6 \lambda) k$, with $k \geq 1$ and non-negative integers $\mu$ and $\lambda$.

Sketch of proof. Since the considered circulant graph on $2 n$ vertices consists of the Ladder graph on $2 n$ vertices as a sugraph plus a set of independent edges, by using an optimal total coloring of the Ladder graph and one new color for the independent set of edges, we obtain the desired total coloring. The technique applied to obtain the total colorings is to construct the circulant graphs by making operations between semigraphs with compatible total colorings. Consider the semigraph $B(n, a)$ with $a$ multiple by $n$, set $n=a \cdot k$ as follows. 
- The vertices of $B(n, a)$ are:

$V=\bigcup_{i=0}^{a-1} V_{i}$, with $V_{i}=\left\{v_{k \cdot i+j} \mid 0 \leq j<k\right\}$.

- The edges of $B(n, a)$ are:

$E=\left(\bigcup_{i=0}^{a-1} E_{i}^{C}\right) \cup\left(\bigcup_{i=0}^{a-2} E_{i}^{R}\right)$, with $E_{i}^{C}=\left\{v_{k \cdot i+j-1} v_{k \cdot i+j} \mid 1 \leq j<k\right\}$ e $E_{i}^{R}=$ $\left\{v_{i \cdot k+j} v_{(i+1) \cdot k+j} \mid 0 \leq j<k\right\}$.

- The semiedges of $B(n, a)$ are partitioned into two classes, called vertical semiedges and horizontal semiedges:

(i) Vertical semiedges: $\left\{v_{i} \cdot \mid 0 \leq i<k\right\} \cup\left\{v_{(a-1) \cdot k+i} \cdot \mid 0 \leq i<k\right\}$, where the sets are called top semiedges and bottom semiedges, respectively, and for $0 \leq i<k$, $v_{i}$. is the $i$-th top semiedge and $v_{(a-1) \cdot k+i}$ is the $i$-th bottom semiedge.

(ii) Horizontal semiedges: $\left\{v_{i \cdot k} \cdot \mid 0 \leq i<a\right\} \cup\left\{v_{(i+1) \cdot k-1} \cdot \mid 0 \leq i<a\right\}$, where the sets are called left semiedges and right semiedges, respectively.

In the following, we present additional definitions and operations that will be used in the construction of total colorings. Given two semiedges $x \cdot$ and $y$, the junction of $x$. with $y$. means replacing $x$. and $y$. by an edge $x y$. The vertical merge between $B(n, a)$ and $B(n, b)$ is the semigraph $B(n+m, a+b)$ obtained by the junction of the bottom semiedges of $B(n, a)$ with the top semiedges of $B(m, b)$, that is, the junction of $v_{(a-1) \cdot k+i}$ of $B(n, a)$ with $v_{i}$. of $B(m, b)$, for $0 \leq i<k$. The horizontal merge between $B(n, a)$ and $B(m, a)$ is the semigraph $B(n+m, a)$ obtained by junction of the right semiedges of $B(n, a)$ with the left semiedges of $B(m, a)$, that is, the junction of $v_{i \cdot k} \cdot$ of $B(n, a)$ with $v_{(i+1) \cdot k-1}$ of $B(m, a)$, for $0 \leq i<a$. Finally, the $p$-closure of $B(n, a)$ is the graph obtained by the junction of the following semiedges: $(i)$ the left semiedges with the right semiedges, that is, for each $0 \leq i<a$, the junction of $v_{i \cdot k+1} \cdot$ with $v_{(i+1) \cdot k} \cdot$; and (ii) the top semiedges with the bottom semiedges, that is, the junction of each $i$-th top semiedge with the $(i-p)$-th bottom semiedge (the positions are taken modulo $k$ ).

We construct 5 -total colorings of the investigated class of graphs by using 5 -total colorings of appropriate copies of semigraphs $B(6,2 k)$ and $B(8,2 k)$. For each $k \geq 1$, by merging semigraphs $B(8,2 k)$ with semigraphs $B(6,2 k)$ (or with copies of the same one) with 5-total colorings and making the closure of the resulting semigraph in a way that the colorings do not conflict in the process, we construct all circulant graphs of Theorem 1 with a 5-total coloring. Figure 2 presents a 5-total coloring of $C_{8}(2,3)$ and the semigraph $B(8,2)$ with a 5-total coloring. By making the 3-closure of this semigraph, we obtain the graph $C_{8}(2,3)$.
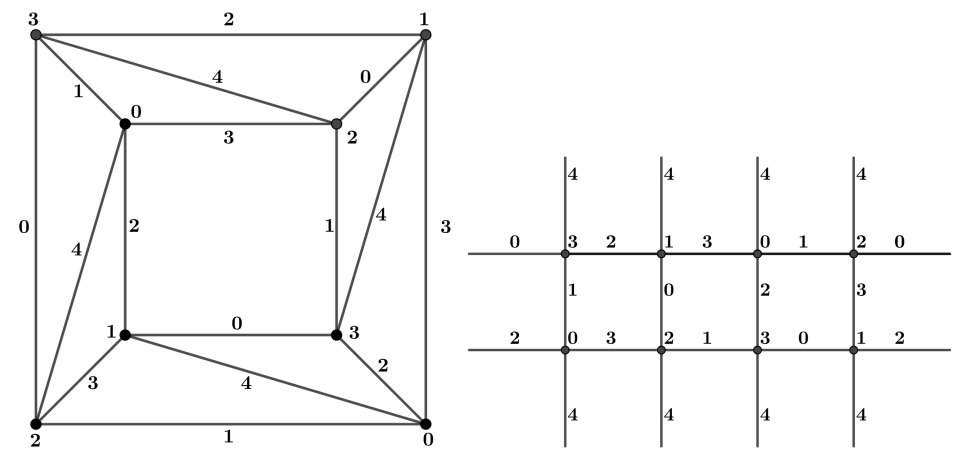

Figure 2. Graph $C_{8}(2,3)$ with a 5-total coloring by using the semigraph $B(8,2)$. 
Another example is presented in the following. By merging semigraphs $B(8,2)$ and $B(6,2)$ and by making the 3-closure of the resulting semigraph with a 5-total coloring, we obtain the graph $C_{14}(2,3)$ with a 5-total coloring, presented in Figure 3.

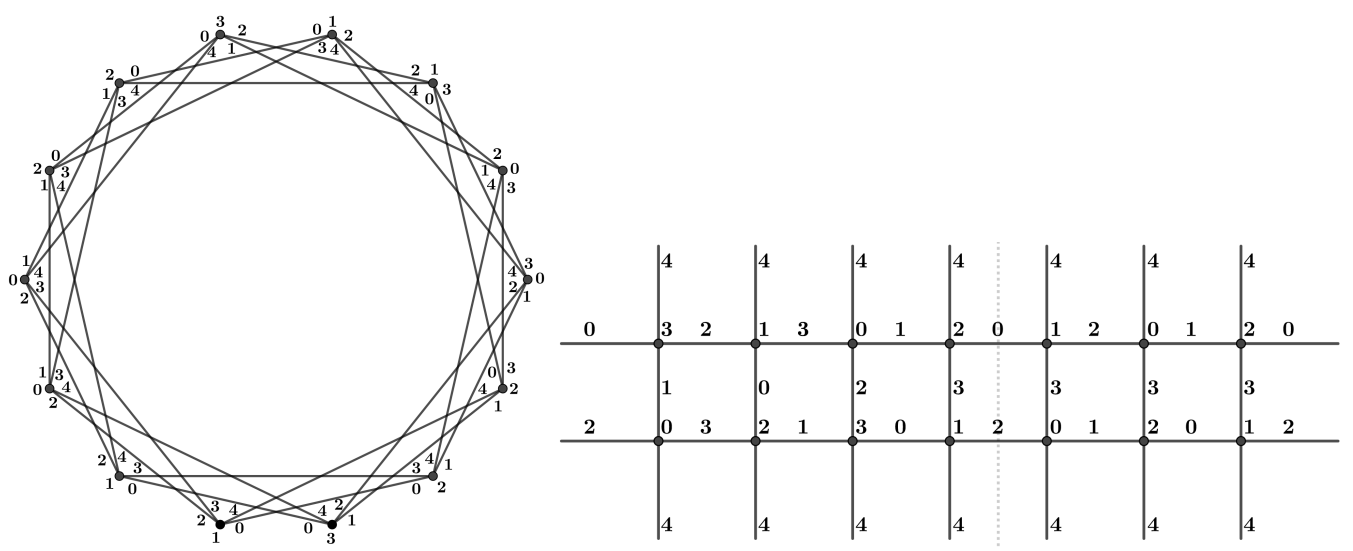

Figure 3. A 5-total coloring of graph $C_{14}(2,3)$ and the horizontal merging between semigraphs $B(8,2)$ and $B(6,2)$.

It is well known that $G(5,1)$ is Type 2 [Chetwynd and Hilton 1988] and we could not determine the Type of $C_{10}(2,3)$. So, from Theorem 1 for $k=1, n \geq 6$ and $n \neq 10$, we conclude this work with the following consequence.

Corollary 1. The circulant graphs $C_{n}(2,3)$, for even $n \geq 6$ and $n \neq 10$, are Type 1 .

\section{References}

Behzad, M. (1965). Graphs and their chromatic numbers. PhD thesis, Michigan University.

Campos, C. (2006). O problema da coloração total em classes de grafos. PhD thesis, University of Campinas, São Paulo.

Chetwynd, A. G. and Hilton, A. J. W. (1988). Some refinements of the total chromatic number conjecture. Congr. Numer., pages 195-216.

Hackmann, A. and Kemnitz, A. (2004). Circular total colorings of cubic circulant graphs. J. Combin. Math. Combin. Comput., pages 65-72.

Khennoufa, R. and Togni, O. (2008). Total and fractional total colourings of circulant grap. Discrete Math., pages 6316-3329.

Vizing, V. (1968). Some unsolved problems in graph theory. Russian Math. Surveys, 23:125-141.

Yap, H. (1996). Total colourings of graphs. Springer, Berlin.

Zorzi, A. (2019). Coloração total em grafos potências de ciclo. Master's thesis, Federal University of Rio de Janeiro. 